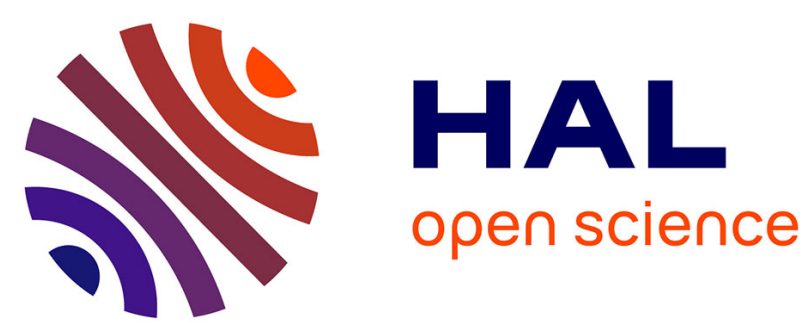

\title{
Synchrotron microbeam radiation therapy induces hypoxia in intracerebral gliosarcoma but not in the normal brain.
}

Audrey Bouchet, Benjamin Lemasson, Thomas Christen, Marine Potez, Claire Rome, Nicolas Coquery, Céline Le Clec'H, Anaick Moisan, Elke Bräuer-Krisch, Géraldine Leduc, et al.

\section{To cite this version:}

Audrey Bouchet, Benjamin Lemasson, Thomas Christen, Marine Potez, Claire Rome, et al.. Synchrotron microbeam radiation therapy induces hypoxia in intracerebral gliosarcoma but not in the normal brain.. Radiotherapy \& Oncology, 2013, 108 (1), pp.143-8. 10.1016/j.radonc.2013.05.013 . inserm-00855558

\section{HAL Id: inserm-00855558 https://www.hal.inserm.fr/inserm-00855558}

Submitted on 29 Aug 2013

HAL is a multi-disciplinary open access archive for the deposit and dissemination of scientific research documents, whether they are published or not. The documents may come from teaching and research institutions in France or abroad, or from public or private research centers.
L'archive ouverte pluridisciplinaire HAL, est destinée au dépôt et à la diffusion de documents scientifiques de niveau recherche, publiés ou non, émanant des établissements d'enseignement et de recherche français ou étrangers, des laboratoires publics ou privés. 


\title{
Synchrotron Microbeam Radiation Therapy induces hypoxia in intracerebral gliosarcoma but not in the normal brain
}

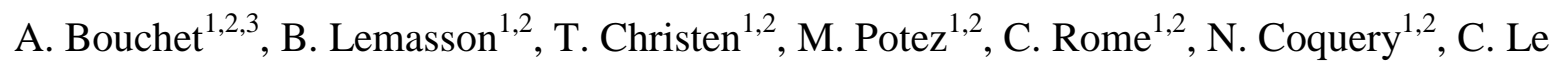 \\ Clec'h $^{3}$, A. Moisan ${ }^{1,2}$, E. Bräuer-Krisch ${ }^{3}$, G. Leduc ${ }^{3}$, C. Rémy ${ }^{1,2}$, J. A. Laissue ${ }^{4}$, E.L.

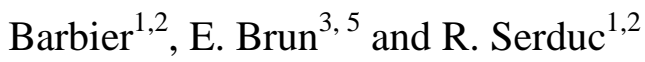 \\ ${ }^{1}$ INSERM, U836, Grenoble, F-38043, France \\ ${ }^{2}$ Université Joseph Fourier, Grenoble Institut des Neurosciences, UMR-S836, Grenoble, F- \\ 38043, France \\ ${ }^{3}$ ESRF, Grenoble, France \\ ${ }^{4}$ Institute of Pathology, University of Bern, Switzerland \\ ${ }^{5}$ Faculty of Physics, Ludwig Maximilians University, 85748 Garching, Germany
}

Key words: Synchrotron Microbeam Radiation Therapy - Brain Tumors - Oxygen Saturation - vessel radiation responses - MRI - Rat 


\section{Abstract \\ Purpose}

Synchrotron microbeam radiation therapy (MRT) is an innovative irradiation modality based on spatial fractionation of a high-dose x-ray beam into lattices of microbeams. The increase in lifespan of brain tumor-bearing rats is associated with vascular damage but the physiological consequences of MRT on blood vessels have not been described. In this manuscript, we evaluate the oxygenation changes induced by MRT in an intracerebral 9L gliosarcoma model.

\section{Methods}

Tissue responses to MRT (two orthogonal arrays $(2 \times 400 \mathrm{~Gy})$ ) were studied using magnetic resonance-based measurements of local blood oxygen saturation $\left(\mathrm{MR}_{-} \mathrm{SO}_{2}\right)$ and quantitative immunohistology of RECA-1, Type-IV collagen and GLUT-1, marker of hypoxia.

\section{Results}

In tumors, MR_SO2 decreased by a factor of 2 in tumor between day 8 and day 45 after MRT. This correlated with tumor vascular remodeling, i.e. decrease in vessel density, increases in half-vessel distances (×5) and GLUT-1 immunoreactivity. Conversely, MRT did not change normal brain MR_SO2, although vessel inter-distances increased slightly.

\section{Conclusion}

We provide new evidence for the differential effect of MRT on tumor vasculature, an effect that leads to tumor hypoxia. As hypothesized formerly, the vasculature of the normal brain exposed to MRT remains sufficiently perfused to prevent any hypoxia. 


\section{Introduction}

Synchrotron microbeam radiation therapy (MRT) uses high radiation doses delivered through near-parallel thin $(25-75 \mu \mathrm{m})$ beams separated by few hundred microns (for review [1]). This unique irradiation modality slows, and sometimes ablates, brain tumors in rodents [2]. The mechanisms underlying MRT efficiency are not fully understood. In a previous study [3], we demonstrated that 9L tumor growth inhibition was correlated to a denudation of the tumor vascular endothelium, leading to significant decreases in tumor blood volume and vessel diameter [3]. This preferential effect of MRT on tumor vessels might induce tumor necrosis through a decrease in perfusion and oxygen supply. On the contrary, normal brain vessels exhibited a high radioresistance to spatially fractionated irradiations [3], even for doses up to $1 \mathrm{kGy}[4,5]$ limited radiation damage occurred in unidirectionally irradiated normal brain tissues $[2,6]$. Australian teams recently suggested a new hypothesis to explain MRT efficiency, stating that heavily irradiated tumor cells ("peak cells") intermixing with sub-lethally exposed cells ("valley cells") may increase tumor control via cell communication [7]. The only molecular response in support of tumoral tissues reacting to MRT according to that hypothesis, to our knowledge, is the description, by Bouchet and colleagues [3], of a high expression of the Vascular Endothelium Growth Factor (VEGF) in brain tumors exposed to MRT, but not in unirradiated tumors.

Increase in VEGF after X-ray exposure is extensively reported in the literature but its role in radiation response remains controversial [8-11]. Radio-induced VEGF originates from different sources, e.g., from endothelial cells, tumor cells, and extracellular matrix. Radical Oxygen Species (ROS) produced by irradiated tissue and hypoxia induce the production of Hypoxia Inducible Factor $1 \alpha(\mathrm{HIF} 1 \alpha)$, which in turn stimulates the expression of angiogenic factors such as VEGF. HIF1 $\alpha$ is also expressed in tumors after radiation-induced vessel necrosis and appears to regulate apoptotic molecular pathways [12]. Monitoring tumor 
hypoxia before and after treatment becomes relevant in patients with glioblastoma since recurrent tumors are often hypoxic [13] and less radiosensitive. Indeed, tumor response to Xrays is linked to local tumor oxygenation [14-16].

In this work, we characterized oxygenation changes induced by MRT on a rat brain model, 9L gliosarcoma (9LGS). We used a recently developed MR method [17] to measure local blood oxygen saturation (MR_SO ${ }_{2}$ ). Evolving changes in blood oxygen saturation were correlated to morphological modifications of the tumor vascular network and to the expression of GLUT-1 (GLUcose Transporter 1, a cellular hypoxia marker [18]), as assessed by immunohistology. 


\section{Methods}

Procedures related to animal care conformed to the Guidelines of the French Government (licenses \#380325/\#380321, authorized labs A3818510002/A3851610008/ A3851610004). Rats were anesthetized with an intraperitoneal injection of xylazine/ketamine (64.5/5.4 mg. $\mathrm{kg}^{-1}$ for tumor implantations and irradiations) and with $2.5 \%$ isoflurane in air for each MRI session.

\section{Tumor implantations and MRT}

9LGS cells $\left(n=10^{4}\right)$ were implanted in rat brains as previously described [19]. Nine days after inoculation, rats underwent an anatomical MR imaging $\left(\mathrm{T}_{2}\right.$ weighted) in order to sort them into 2 groups of similar tumor size. On day 10 (D10), one group of rats was irradiated in microbeam mode at the European Synchrotron Radiation Facility (ID17, Grenoble, France). The detailed irradiation setup is described in [3]. Briefly, the synchrotron $\mathrm{X}$-rays beam was collimated into a lattice of parallel, $50 \mu \mathrm{m}$-wide, microbeams separated on center by $200 \mu \mathrm{m}$. Two arrays of 40 microbeams, each $10 \mathrm{~mm}$-high and $\approx 8 \mathrm{~mm}$-wide, one anteroposterior, one lateral, were crossfired at the center of the tumor with an entrance dose of 400 Gy each. The peak and valley doses were 350/700 Gy and 12.5/25 Gy in the unidirectionally/bidirectionally irradiated parts of the brain, respectively, as in the previous study [3].

\section{MR_SO 2 Measurements}

MRI was performed at $4.7 \mathrm{~T}$ (Avance III console; Bruker) of the Grenoble MRI facility IRMaGe. Individual blood oxygen saturation maps were obtained in all rats listed under "MRI" in table 1, using a multi-parametric quantitative Blood Oxygen Level Dependent (BOLD) approach. This protocol, fully detailed in [17], uses several MR sequences to extract 
the oxygenation information from a baseline $\mathrm{T} 2 *$ weighted signal. The MR protocol duration was about 30 min per animal for a spatial resolution of $234 \times 234 \times 1000 \mu \mathrm{m}^{3}$.

Blood samples of each rat were collected via the tail vein before and after each MRI session and examined in a blood gas analyzer (ABL 510, Radiometer, Copenhagen, Denmark). The MR_SO $\mathrm{SO}_{2}$ maps of each unirradiated rat and rats exposed to MRT (table 1, "MRI") were superimposed on $\mathrm{T}_{2}$-weighted MRI images acquired at different times after 9LGS implantation (Fig. 1a). To obtain the mean values shown in Fig. 1 b, we calculated the mean pixel value of $\mathrm{MR} \_\mathrm{SO}_{2}$ per individual rat in tumors and contralateral tissues. The mean values (of the mean pixel values obtained for individual rats) obtained for each group at different time points were plotted versus time (Fig. 1b) and compared by use of two way ANOVA Bonferroni post-tests.

\section{Histological procedures and quantitative immunohistology}

\section{Vessel examination and hypoxia quantifications}

The evolution of vessel morphology and GLUT-1 expression were analyzed on, $18 \mu \mathrm{m}$-thick frozen sections of brains sampled at D9, D18 and D55 after implantation. Briefly, after fixation and saturation, brain sections were incubated overnight at $4{ }^{\circ} \mathrm{C}$ with primary antibodies (anti Type-IV collagen, (SouthernBiotech F5205-N795, 1/1000), anti RECA-1 (AbDserotec MCA970R, 1/300) and anti GLUT-1 (ThermoScientific PA5-16793, 1/500)). Sections were washed and exposed to the secondary antibodies for $2 \mathrm{~h}$ at room temperature. The sections were examined with a Nikon Eclipse E600 microscope equipped for epifluorescence.

Image analysis was performed using a home-made macro on ImageJ. After band pass filtering, colocalization maps were built using images corresponding for Type-IV collagen 
and RECA-1 reactivity. The resulting images were pre-filtered (median) and segmented using an automatic thresholding technique [20]. The voronoi diagrams were calculated on the ultimate erodes of the vessels. The number of voronoi cells gave the number of vessels. The half distances between vessels (hDV) were derived from the resulting voronoi images. GLUT1 positive areas (GLUT-1+) were manually delineated on microscopic images and expressed in percentage of the total surface images. The means (per experimental group and day after inoculation) of hDV, vessel densities and GLUT-1+ were compared using a non parametric Mann-Whitney test (significant for $\mathrm{p}<0.05$ ). 


\section{Results}

\section{MRT reduced tumor oxygenation - MRI evaluation}

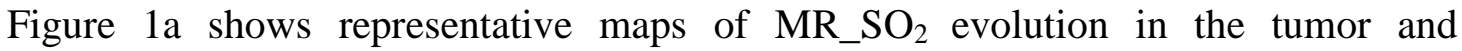
contralateral hemisphere of unirradiated and of irradiated rats. The percentages of $\mathrm{MR}_{-} \mathrm{SO}_{2}$ (means $\pm \mathrm{SD}$ ) in tumor and contralateral hemispheres are displayed in figure $1 \mathrm{~b}$. There were no significant changes in MR_SO ${ }_{2}$ during $9 \mathrm{LGS}$ growth in unirradiated rats (mean $\mathrm{MR}_{-} \mathrm{SO}_{2}$ : $72.0 \pm 9.9 \%$ (D9) and 70.2 $\pm 3.2 \%$ (D18)). MRT induced a progressive and significant decrease of tumor MR_SO 2 starting on D18 after MRT (from $75.1 \pm 7.9 \%$ (D9) to $60.6 \pm 5.6 \%$ (D18), $\mathrm{p}=0.046$ compared with values in unirradiated rats, Fig. 1b). Blood oxygen saturation in individual tumors continued to decrease until the last measurement point (D55), reaching $34.8 \pm 11.3 \%$ ( $<<0.001$ compared with MRT group on D18). No significant changes of MR_SO $\mathrm{SO}_{2}$ occurred in contralateral brain tissues of unirradiated rats (D9 - D18) nor of rats exposed to MRT (D9 - D55) during the whole experiment, although there was a slight decline around D25 in irradiated rats.

\section{MRT-induced MR_SO 2 reduction correlates with endothelial denudation in tumors}

In Figure 1c, we plotted the MR_SO $\mathrm{SO}_{2}$ evolution in the tumor of rats exposed to MRT with respect to tumor Type-IV collagen/RECA-1 colocalization fraction calculated by Bouchet et al. 2010 [3]. The two parameters correlate significantly (linear fit $\mathrm{r}^{2}=0.91$, Standard-Deviation of Estimate (SDE): 0.07). 
MRT induced tumor vessel spatial redistribution, increased vessel inter-distances and

\section{GLUT-1 overexpression}

\section{Contralateral hemisphere, unidirectionally irradiated}

Whatever the observation delay, RECA-1 and GLUT-1 were expressed on endothelial cells and were detected in every Type-IV collagen positive entity (not shown). Slight changes in contralateral vessel morphology were observed during MRT. Indeed, the hDV (Fig. 2a), measured between RECA-1/Type-IV collagen positive structures in the unidirectionally irradiated slice of the contralateral hemisphere in the MRT group augmented from $25.8 \pm 8.6$ to $33.7 \pm 16.2 \mu \mathrm{m}$ (D9 and D55, respectively) ( $\mathrm{p}<0.001)$ while no changes were detected in corresponding hemispheres of unirradiated animals. Vessel density decreased significantly during the whole experiment in unidirectionally irradiated contralateral tissues (MRT group: $135 \pm 15,110 \pm 22$ vessels per field of view on D9 and D55 after implantation, respectively, p<0.05, Fig. 2c).

\section{Unirradiated 9LGS}

Figure 3 displays Type-IV collagen, RECA-1 and GLUT-1 pattern evolutions in unirradiated rats and rats exposed to MRT. As observed in normal tissue, each tumor vessel was positive for RECA, GLUT-1 and Type-IV collagen in unirradiated animals. Morphologic changes were observed (Fig. 3) and quantified (Fig. 2) during tumor growth: (i) the mean half distance between vessels (hDV) significantly increased from $35.4 \pm 13.2$ to $42.6 \pm 31.2 \mu \mathrm{m}$ between D9 and D18 ( $<<0.001$, Fig. $2 b$ ) and (ii) the vessel density decreased from $86 \pm 24$ to $40 \pm 19$ vessels per FOV between D9 and D18 (p<0.001, Fig. 2d). 


\section{Irradiated 9LGS}

The effects of MRT on tumor vessels are shown on figure 3. The density of tumor vessels assessed by Type IV collagen labeling was progressively reduced with the delay after MRT: from D18 the proportion of RECA-1/GLUT-1 positive entities decreased. Consequently, the mean $\mathrm{hDV}$ at $\mathrm{D} 18$ increased from $42.6 \pm 31 \mu \mathrm{m}$ in unirradiated tumors to $86.9 \pm 83.4 \mu \mathrm{m}$ in tumors exposed to MRT ( $<<0.001$, Fig. $2 \mathrm{~b}$ ). The median $\mathrm{hDV}$ were $33.8 \mu \mathrm{m}$ and $67.6 \mu \mathrm{m}$ respectively. $\mathrm{hDV}$ continued to increase until $151.4 \pm 143.5 \mu \mathrm{m}$ on D55 ( $<<0.001$ compared with D18, Fig. 2b). Vessel density was significantly reduced in tumors exposed to MRT compared with unirradiated tumors on D18 and further decreased until D55 ( $<<0.05$ and $\mathrm{p}<0.001$, respectively, Fig. 2d). GLUT-1 immunoreactivity was observed in some tumor vessels but also in tumor cells distant from RECA-1-positive vessels (Fig. 3). In the MRT group, the percentage of GLUT-1 positive tumor areas increased with the delay after irradiation (Fig. 2e). Eight days after MRT (D18), 16.4\% of the tumor area was GLUT-1 positive, versus $5.6 \%$ in tumors of the time matched unirradiated group. This proportion reached $27.4 \%$ on D25 and $85.1 \%$ on D55. When $\mathrm{MR}_{-} \mathrm{SO}_{2}$ is plotted against GLUT-1 positive areas (Fig. 1d), the two parameters correlated with a $R^{2}=0.82$ (exponential fit). 


\section{Discussion}

We studied the effects of MRT on the oxygenation and the vascular networks of 9LGS. We showed that microbeam irradiation induced a significant decrease in tumor $\mathrm{MR} \_\mathrm{SO}_{2}$ but not in the unidirectionally irradiated coronal slice of the normal hemisphere. This reduction in $\mathrm{MR} \_\mathrm{SO}_{2}$ strongly correlated with endothelial cell rarefaction and increased inter-vessel distances. The progressive diminution in oxygenation induced tumor hypoxia. The latter was confirmed by GLUT-1 overexpression which non-linearly correlated with the MR_SO $\mathrm{SO}_{2}$ parameter. These results give new evidence for a preferential effect of MRT on tumor vessels versus normal vessels, and a novel insight in biological/physiological tumor responses to MRT.

In the last decades, MRI appeared as a powerful tool for mapping brain tumors in clinical cancer diagnosis and treatment response. MRI allows the measurements of multiple physio/pathological parameters of tumors, such as cellularity, necrosis, blood volume, vessel size index, vessel permeability. The present study showed that, $\mathrm{MR}_{-} \mathrm{SO}_{2}$ measurements also give some information about brain tumor hypoxia since a strong correlation was found between MR_SO 2 and GLUT-1 expression. An analogous evaluation of the MR estimates of tissue oxygenation has been described in another study on gliosarcoma treated by an antiangiogenic drug [21]. Furthermore, our results suggest that $\mathrm{MR}_{-} \mathrm{SO}_{2}$ depicted tumor vessel regression after MRT. Indeed, we calculated a 0.91 correlation coefficient between MR_SO $\mathrm{SO}_{2}$ levels and endothelial cell rarefaction in irradiated tumors. Such correlation had not been found in unirradiated tumors [22]. This observation might have a clinical impact since MR_SO $\mathrm{SO}_{2}$ measurements alone would help in the evaluation of glioma radiation response by describing, non-invasively, multiple vascular and physiological parameters which were until now only discernible by immunohistologic studies. 
Several studies gave evidence for normal brain tissue resistance to spatially fractionated irradiations $[1,2,6]$. This has been attributed to the particular radio-tolerance of normal vascular networks which supply nutrients to exposed cerebral tissue $[4,5]$ and reduce radiation-induced hypoxia / ischemia. In this study, we show that despite an increase in vessel inter-distances $(+8 \mu \mathrm{m})$ and a decrease in vessel density $(\sim 18 \%)$ in the subacute stage after MRT (45 days post irradiation), irradiated tissues in the contralateral hemisphere remained homogenously and sufficiently perfused. Indeed, mean inter-vessel distances measured in unidirectionally irradiated contralateral rat brains $(25.8 \pm 8.6$ to $33.7 \pm 16.2 \mu \mathrm{m})$ remained in the range of the one given for normal human grey matter, i.e. around $32 \mu \mathrm{m}$ [23]. As shown by Bouchet et al.[3] and confirmed in this study, normal brain capillaries exposed to X-ray microbeams are still immunoreactive for RECA-1 and GLUT-1 thus proving the presence of endotheliocytes after MRT. The integrity of the endothelium maintains an adequate supply in oxygen and nutrients to unidirectionally irradiated tissues in the contralateral hemisphere and precludes hypoxia whatever the delay after irradiation. Furthermore, the structural modifications of the normal vascular network are not surprising since the radiation dose delivered in the present study was undeniably high enough to induce endothelial cell death and vascular remodeling $[24,25]$. These changes matched the previously shown maximum overexpression of VEGF in contralateral hemispheres on D55 but our previous work showed that VEGF could be detected by western blot as soon as 8 days after MRT[3]. Because in unidirectionally irradiated parts of the contralateral hemisphere we observed (i) no changes of the MR_SO $\mathrm{S}_{2}$, (ii) only minor morphometric changes of vessels, (iii) no cerebral cells other than endothelial cells displaying GLUT-1 labeling and (iv) because GLUT-1 is regulated by HIF-1 $\alpha$ [26], the overexpression of VEGF after MRT was presumably not related to tissular hypoxia. The dose dependent VEGF production in normoxia after radiation has been documented in different cell lines $[10,11,27]$. Brieger et al. hypothesised that the release of 
VEGF protects the endothelium thereby increasing its radiation resistance [11]. The in vivo source of VEGF after MRT remains unknown but VEGF might help in protecting normal tissue from the effects of MRT. Indeed, no drastic changes and no major damage were observed in normal brain vessels in HE-stained sections of unidirectionally irradiated rat brains [3].

Our previous study showed that identical irradiation of 9LGS leads to $80 \%$ tumor control on D55 after irradiation [3]. The present follow-up study current gives more insight into physiological mechanisms involved in tumor response to MRT; the results confirm the differential effect exerted by MRT on tumor vessels compared to normal vessels. Indeed, MRT induced a progressive hypoxia of the gliosarcoma, starting one week after irradiation. This was associated with a significant decrease in the number of tumor vessels and a significant increase in tumoral intercapillary distances. On D18, half of the intervessel distances were wider than $\sim 134 \mu \mathrm{m}$ in irradiated tumors while unirradiated tumors exhibited median intervessel distances of $\sim 66 \mu \mathrm{m}$. Our observations are in accordance with previous results (between 42.5 and $105 \mu \mathrm{m}$ [28]) measured in 9LGS. Median distances between tumor vessels even reached $\sim 246 \mu \mathrm{m}$ on D55 while the maximum oxygen diffusion limit is $100 \mu \mathrm{m}$ according to Carmeliet and Jain [29]. MRT caused a spatial redistribution of tumor vessels that probably led to inhomogeneous perfusion of the tumor. Indeed, long inter-vessel distances cannot warrant an adequate diffusion of oxygen to tumor cells [29] thus, MRT induced local hypoxia as detected on D55 by the presence of distinct areas of GLUT-1 positive tumor cells. The decrease in oxygen saturation was linearly related to endothelial cell rarefaction in tumor vessels and indirectly to the decrease in tumor blood volume (Fig. 1 and [3]). Altogether, our last studies characterize new relevant tumoricidal mechanisms of MRT. We assume that a three-step process might describe the tumor response to X-ray microbeam irradiation. During the first week after exposure, after a G2/M blockade [30], the tumor 
progressively shrinks due to radiation-induced mitotic cell death, pyknosis and abnormal replicative processes such as endoreplication and aberrant mitoses [30]. In the meantime, ionizing radiation also induces tumor endothelium denudation, but blood supply is still maintained via damaged and permeable vessels [3]. During the first week after irradiation, MRT increases VEGF production and causes vascular remodeling, doubling the distance between tumor vessels. From D18 on, the significant decrease in tumor blood volume [3] induces hypoxia in 9LGS exposed to MRT, as shown by the increasing GLUT-1 immunolabeling. The low standard deviations obtained for any measurement performed until D25, i.e., 15 days after MRT, show that the behavior of all the tumors was comparable in terms of tumor size, blood volume, vessel size, $\mathrm{MR} \_\mathrm{SO}_{2}$, vessel denudation and VEGF expression (see [3]). However, despite this homogenous early response, 1 tumor out of 5 studied on D55 recurred [3]. The D25-D55 delay range appears critical for tumor outcome; a non-coplanar re-irradiation [19] delivered at this time might be instrumental in killing resistant tumor cells responsible for the recurrence. Nevertheless, our study demonstrates that the additional biological mechanisms that occur in the sub-acute stage after exposure determine tumor outcome after MRT. These processes have to be identified; the role of radiation-induced hypoxia in tumor and its biological implication in recurrence needs to be studied in detail: the treatment of recurrent hypoxic tumors might be problematic because of their high radio- and chemo-resistance [27, 31]. Depending on the extent of the radiationinduced hypoxia, different and even opposite effects on cell survival/cell death could be observed: tumor cells can either adapt to acute and mild hypoxia or initiate apoptosis in case of severe hypoxia [32]. 
Conflict of interest: none.

Acknowledgments: The authors thank the "Conseil Régional Rhône-Alpes", la "Ligue contre le Cancer, comité de la Drome", "l'Association pour la Recherche contre le Cancer" and Imoxy ANR for research funding.

Reprint requests: Raphaël Serduc. INSERM U836. ESRF, 6 rue Horowitz, F38043 Grenoble, France. Tel.: 0033438881960. Fax: 0033476882825 E-mail: raph.serduc@gmail.com 


\section{References}

[1] Brauer-Krisch E, Serduc R, Siegbahn EA, et al. Effects of pulsed, spatially fractionated, microscopic synchrotron X-ray beams on normal and tumoral brain tissue. Mutat Res. 2010;704:160-166.

[2] Laissue JA, Geiser G, Spanne PO, et al. Neuropathology of ablation of rat gliosarcomas and contiguous brain tissues using a microplanar beam of synchrotron-wiggler-generated $\mathrm{X}$ rays. Int J Cancer. 1998;78:654-660.

[3] Bouchet A, Lemasson B, Leduc G, et al. Preferential effect of synchrotron microbeam radiation therapy on intracerebral 9L gliosarcoma vascular networks. Int. J. Rad. Onc. Biol. Phys. 2010;78:1503-1512.

[4] Serduc R, Christen T, Laissue J, et al. Brain tumor vessel response to synchrotron microbeam radiation therapy: a short-term in vivo study. Phys Med Biol. 2008;53:3609-3622.

[5] Serduc R, Verant P, Vial JC, et al. In vivo two-photon microscopy study of short-term effects of microbeam irradiation on normal mouse brain microvasculature. Int J Radiat Oncol Biol Phys. 2006;64:1519-1527.

[6] Laissue JA, Blattmann H, Wagner HP, et al. Prospects for microbeam radiation therapy of brain tumours in children to reduce neurological sequelae . Dev Med Child Neurol. 2007;49:577-581.

[7] Crosbie JC, Anderson RL, Rothkamm K, et al. Tumor cell response to synchrotron microbeam radiation therapy differs markedly from cells in normal tissues. Int J Radiat Oncol Biol Phys. 2010;77:886-894.

[8] Kim JH, Chung YG, Kim CY, et al. Upregulation of VEGF and FGF2 in normal rat brain after experimental intraoperative radiation therapy. J Korean Med Sci. 2004;19:879-886.

[9] Hovinga KE, Stalpers LJ, Van Bree C, et al. Radiation-enhanced vascular endothelial growth factor (VEGF) secretion in glioblastoma multiforme cell lines--a clue to radioresistance? J Neurooncol. 2005;74:99-103.

[10] Brieger J, Schroeder P, Gosepath J, et al. VEGF-subtype specific protection of SCC and HUVECs from radiation induced cell death. Int J Mol Med. 2005;15:145-151.

[11] Brieger J, Schroeder P, Gosepath J, et al. Vascular endothelial growth factor and basic fibroblast growth factor are released by squamous cell carcinoma cells after irradiation and increase resistance to subsequent irradiation. Int J Mol Med. 2005;16:159-164.

[12] Magnon C, Opolon P, Ricard M, et al. Radiation and inhibition of angiogenesis by canstatin synergize to induce HIF-1alpha-mediated tumor apoptotic switch. J Clin Invest. 2007;117:1844-1855. 
[13] Kioi M, Vogel H, Schultz G, et al. Inhibition of vasculogenesis, but not angiogenesis, prevents the recurrence of glioblastoma after irradiation in mice. J Clin Invest. 2010;120:694705.

[14] Moeller BJ, Cao Y, Li CY, et al. Radiation activates HIF-1 to regulate vascular radiosensitivity in tumors: role of reoxygenation, free radicals, and stress granules. Cancer Cell. 2004;5:429-441.

[15] Zhao D, Constantinescu A, Chang C-H, et al. Correlation of tumor oxygen dynamics with radiation response of the dunning prostate R3327-HI tumor. Radiation research. 2003;159:621-31.

[16] Fyles AW, Milosevic M, Wong R, et al. Oxygenation predicts radiation response and survival in patients with cervix cancer. Radiotherapy and oncology $\square:$ journal of the European Society for Therapeutic Radiology and Oncology. 1998;48:149-56.

[17] Christen T, Lemasson B, Pannetier N, et al. Evaluation of a quantitative bold approach to map local blood oxygen saturation. . NMR in Biomedicine. 2011;24:393-403.

[18] Le QT, Courter D. Clinical biomarkers for hypoxia targeting. Cancer Metastasis Rev. 2008;27:351-362.

[19] Serduc R, Brauer-Krisch E, Bouchet A, et al. First trial of spatial and temporal fractionations of the delivered dose using synchrotron microbeam radiation therapy. $\mathrm{J}$ Synchrotron Radiat. 2009;16:587-590.

[20] Otsu N. A threshold selection method from gray-level histograms. IEEE Transactions on Systems, Man and Cybernetics. 1979;9:62 - 66.

[21] Lemasson B, Christen T, Serduc R, et al. Evaluation of the Relationship between MR Estimates of Blood Oxygen Saturation and Hypoxia: Effect of an Antiangiogenic Treatment on a Gliosarcoma Model. Radiology. 2012;265:743-52.

[22] Christen T, Lemasson B, Pannetier N, et al. Is T2* enough to assess oxygenation? Quantitative blood oxygen level-dependent analysis in brain tumor. Radiology.

2012;262:495-502.

[23] Mintun MA, Lundstrom BN, Snyder AZ, et al. Blood flow and oxygen delivery to human brain during functional activity: theoretical modeling and experimental data. Proc Natl Acad Sci U S A. 2001;98:6859-6864.

[24] Lyubimova N, Hopewell JW. Experimental evidence to support the hypothesis that damage to vascular endothelium plays the primary role in the development of late radiationinduced CNS injury. Br J Radiol. 2004;77:488-492.

[25] Lyubimova N V, Coultas PG, Yuen K, et al. In vivo radioprotection of mouse brain endothelial cells by Hoechst 33342. Br J Radiol. 2001;74:77-82.

[26] Harris AL. Hypoxia--a key regulatory factor in tumour growth. Nat Rev Cancer. 2002;2:38-47. 
[27] Lund EL, Hog A, Olsen MW, et al. Differential regulation of VEGF, HIF1alpha and angiopoietin-1, -2 and -4 by hypoxia and ionizing radiation in human glioblastoma. Int $\mathbf{J}$ Cancer. 2004;108:833-838.

[28] Yoshii Y, Sugiyama K. Intercapillary distance in the proliferating area of human glioma. Cancer Res. 1988;48:2938-2941.

[29] Carmeliet P, Jain RK. Angiogenesis in cancer and other diseases. Nature. 2000;407:249257.

[30] Bouchet A, Boumendjel A, Khalil E, et al. Chalcone JAI-51 improves efficacy of synchrotron microbeam radiation therapy of brain tumors. J Synchrotron Radiat.

2012;19:478-482.

[31] Cuisnier O, Serduc R, Lavieille JP, et al. Chronic hypoxia protects against gammairradiation-induced apoptosis by inducing bcl-2 up-regulation and inhibiting mitochondrial translocation and conformational change of bax protein. Int J Oncol. 2003;23:1033-1041.

[32] Greijer AE, Van der Wall E. The role of hypoxia inducible factor 1 (HIF-1) in hypoxia induced apoptosis. J Clin Pathol. 2004;57:1009-1014. 
Figure 1: MRT induced MR_SO2 decrease in intracerebral 9LGS tumors which correlates with endothelium denudation and GLUT-1 overexpression. a- $\mathrm{MR} \_\mathrm{SO}_{2}$ maps superimposed on $\mathrm{T}_{2}$-weighted MRI images acquired in unirradiated rats and rats exposed to MRT at different delays after 9LGS implantation. b- MR_SO $\mathrm{SO}_{2}$ evolution in unirradiated and irradiated 9LGS, in unirradiated contralateral hemispheres, and in tissues of contralateral hemispheres exposed to MRT, at different delays after tumor inoculation. MRT was performed at D10 after implantation. Significantly different from D9 in the same group, $* \mathrm{p}<0.01, * * \mathrm{p}<0.001$, two way ANOVA Bonferroni post-tests. $\mathrm{c}$ and $\mathrm{d}$ - Correlation plots between MR_SO 2 , RECA-1/Type-IV collagen fraction (a) and GLUT-1 positive areas $(\%, b)$ in MRT irradiated 9LGS. SDE: Standard Deviation of Estimate.

Figure 2: MRT increases tumor intervessel distances, reduces tumor vessel density and induces hypoxia in 9LGS. Distribution of half distances between vessels positive for RECA1/Type-IV collagen, derived from Voronoi cells, measured in normal contralateral hemispheres of unirradiated rats (a) and tumoral tissues of rats exposed to MRT (9LGS) (b). Mean number $( \pm$ SEM) of vessels per field of view (FOV) measured in normal contralateral hemispheres of unirradiated (black bars) or irradiated (white bars) rats (c) and tumoral tissues (9LGS) (d) of unirradiated rats (black bars) or rats exposed to MRT (white bars). ePercentage of GLUT-1 positive areas measured in tumors of both unirradiated rats (black bars) and rats exposed to MRT (white bars).

Figure 3: MRT induces tumor vascular remodeling. MRT induces tumor vascular remodeling. Temporal immunohistological follow-up of unirradiated or irradiated normal and tumoral brain vessels. MRT $=$ microbeam radiation therapy. Type IV collagen (blue) and RECA-1 (red) immunolabeling observed in tumors at different times ( $\mathrm{D}=$ days after 
implantation, $\mathrm{T}=$ days before and after MRT). Voronoi diagrams were calculated and represented on merged RECA-1/Type-IV collagen images (merge + vor). Temporal evolution of GLUT-1 expression is shown in green on merged RECA-1/Type-IV collagen images. Scale bar: $200 \mu \mathrm{m}$.

Table 1: Number of tumor-bearing animals studied by MRI and quantitative immunohistology in unirradiated (control) and MRT-treated groups of rats. " $D$ " denotes the days after tumor implantation (on day 0), "T" the days before or after MRT (on day 10). N.a. $=$ not applicable, as no rats survived. 


\begin{tabular}{cc|c|c|c|c|c|c}
\multirow{2}{*}{ MRI } & $\mathrm{D}_{9} \mathrm{~T}_{-1}$ & $\mathrm{D}_{12} \mathrm{~T}_{2}$ & $\mathrm{D}_{15} \mathrm{~T}_{15}$ & $\mathrm{D}_{18} \mathrm{~T}_{8}$ & $\mathrm{D}_{25} \mathrm{~T}_{15}$ & $\mathrm{D}_{55} \mathrm{~T}_{45}$ \\
\cline { 2 - 9 } & Unirradiated & 6 & 8 & 8 & 6 & n.a. & n.a. \\
\hline \multirow{2}{*}{ IMRT } & 4 & 7 & 8 & 7 & 8 & 5 \\
\hline MRT & 4 & 3 & 3 & 3 & 3 & 3
\end{tabular}


a

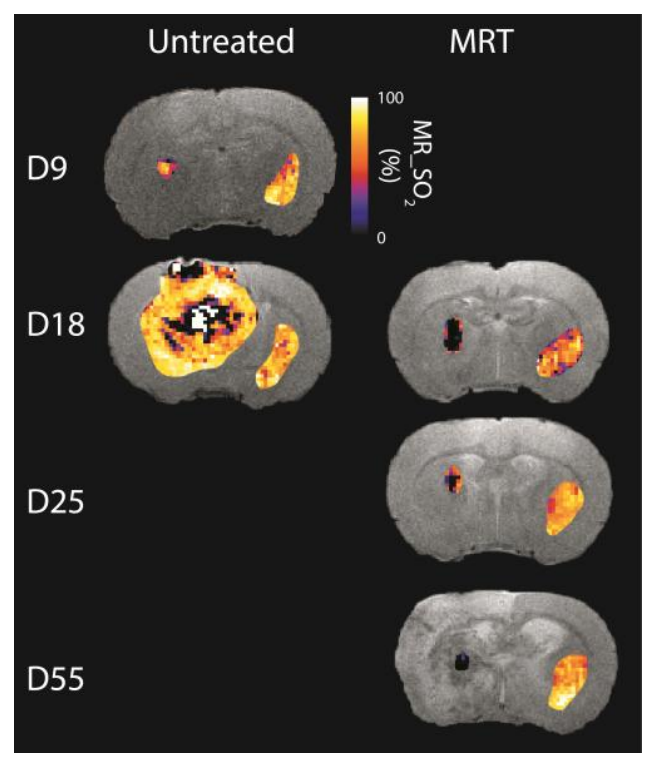

c

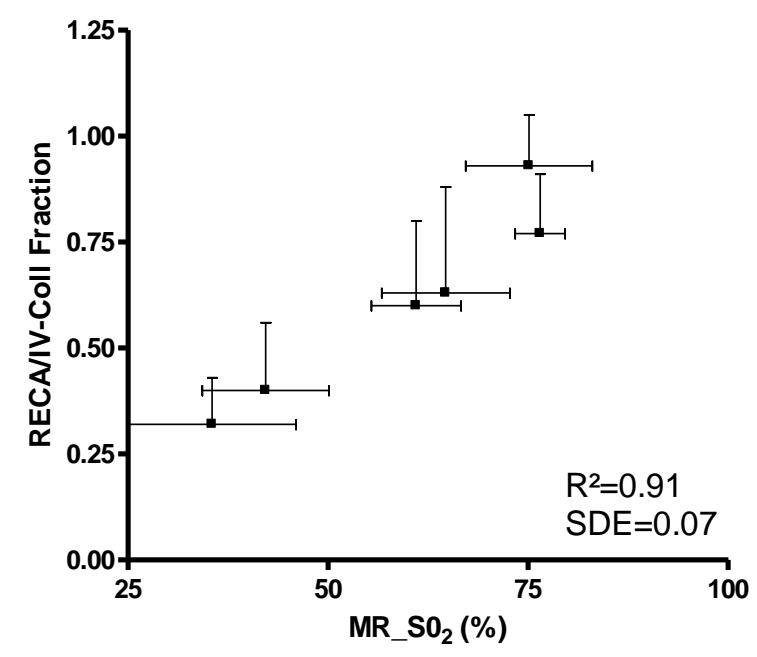

b

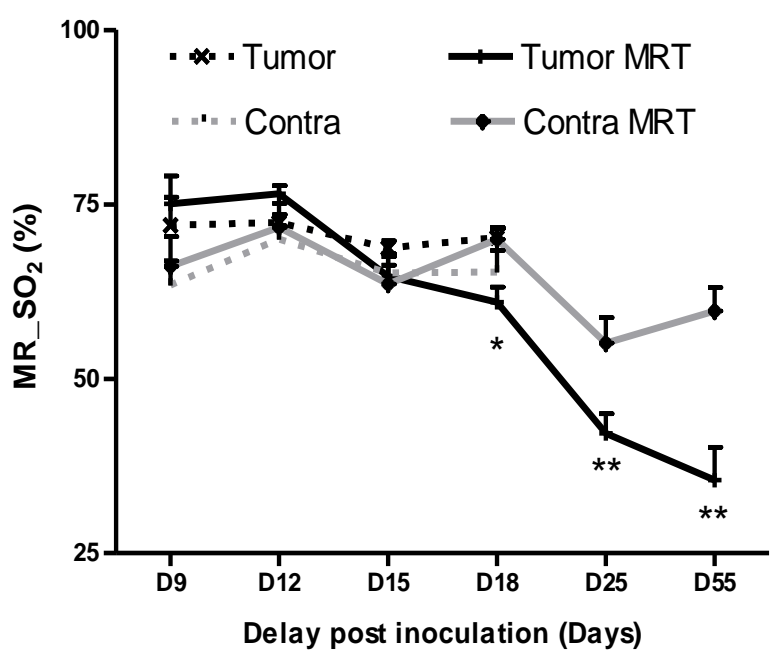

d

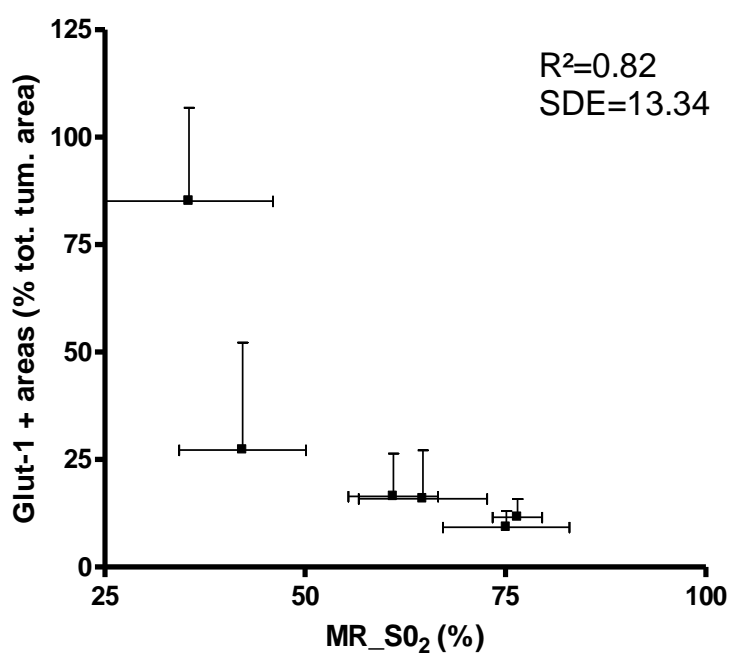



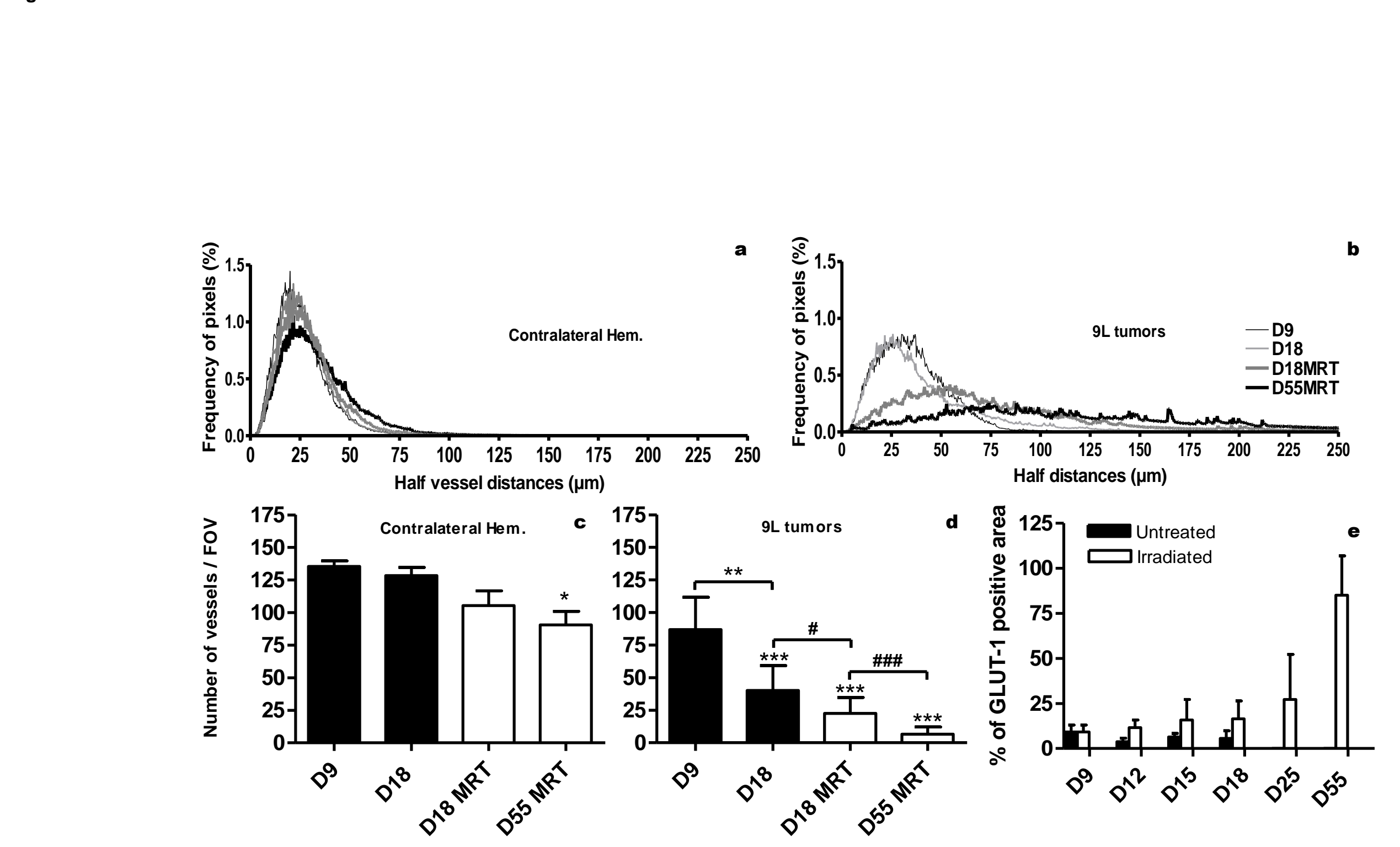

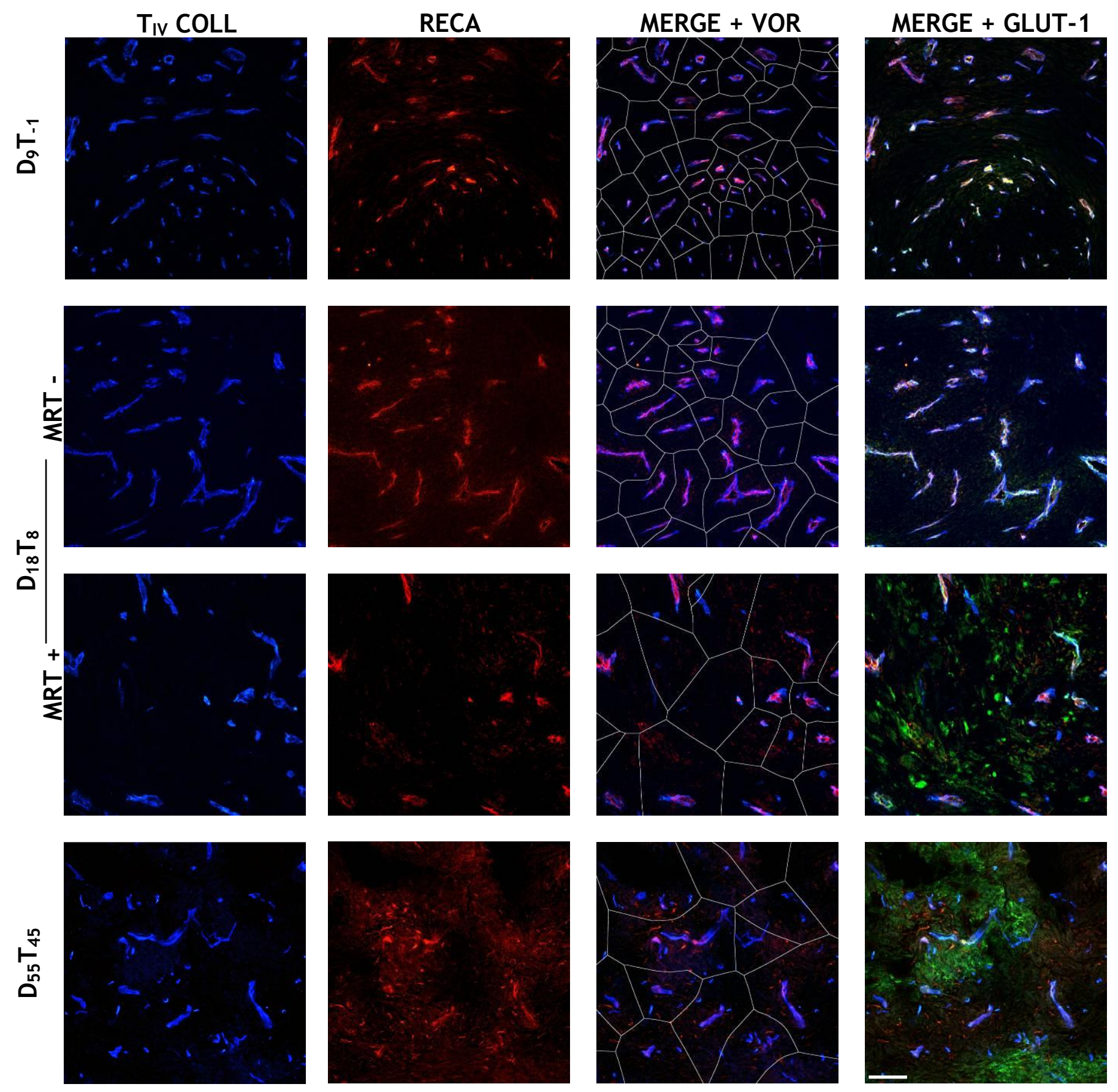